\title{
SUPERNOVAE: WHERE AND WHY DO THEY BREAK OFF?
}

\section{HERRERA}

Departamento de Física, Facultad de Ciencias, Universidad Central de Venezuela, Caracas, Venezuela and L. A. NÚNEZZ

Laboratorio de Física Teórica, Departamento de Física, Facultad de Ciencias, Universidad de Los Andes, Mérida, Venezuela

(Received 21 March, 1991)

\begin{abstract}
The precise mechanism whereby gravitational collapse leads to a type II supernova event is one of the most controversial points in the understanding of the final stages of stellar evolution. The "bounceshock' mechanism and the proposed 'long-term neutrino mediated' processes compete to explain the ejection of the outer envelopes of the star. Despite their differences, both descriptions consider the shock as mainly responsible for the ejection of the outer mantle in a supernova burst. In this note we discuss results from collapse calculations in which the shock is considered as an interface separating the quasi-static stiff core from the outer mantle. In the models considered the shock fades out and becomes a new boundary surface delimiting the compact homogeneous remnant. The resulting pictures become intelligible in the light of a recently proposed generalization of the concept of adiabatic index for systems where radiation flux is present.
\end{abstract}

In the past two decades a large number of different techniques have been introduced to study both the Newtonian and the general relativistic gravitational collapse but, in spite of detailed modeling, considerable theoretical achievements and fruitful discussions, there are still outstanding open questions concerning the precise mechanisms by which core implosions lead to stellar explosions. With the discovery of the supernova SN 1987A there has been a renewed interest in the problem of gravitational collapse of massive stars $\left(\mathscr{M}_{*} \geq 8 \mathscr{M}_{\odot}\right)$. For the first time a recorded neutrino profile provides a wealth of observational data about the nature of the processes producing the final compact remnant. Concerning the gravitational collapse, two points emerge particularly controversial. On one hand, it is not clear enough whether a prompt (cf. Colage and Johnson, 1960; Bethe et al., 1979) a long-term neutrino-mediated (Wilson, 1985; Bethe and Wilson, 1985; Burrows, 1987) or any other alternative mechanism is to be considered in collapse that results in a type II supernova event. On the other hand, interesting polemic have been raised over both the form of the detected neutrino outburst profile, and the possible hydrodynamic scenarios compatible with this detection. The discrepancies between the data collected by KAMIOKANDE II (Hirata et al., 1987) and Irvine-Michigan-Brookhaven (IMB) (Bionta et al., 1987) groups and those reported by the Mont Blanc detector (Aglietta et al., 1987) have motivated a fruitful debate over the specific contour that might represent the detected burst (cf. De Rújula, 1987). The hydrodynamic pictures proposed to be consistent with this first observation range from the prompt type II supernova (Baron et al., 1987; Bruenn, 1987; Kahana 
et al., 1987) and the advective overturn detonation mechanism (Arnett, 1987) to the two-bang explosion yielding either a black hole (Aglietta et al., 1987), a neutron star (De Rújula, 1987), or disruption of a light neutron star in a ultra-close binary system (Stella and Treves, 1987). Despite that, presently it seems to be understood some of the details for SN 1987A event (cf. Trimble, 1988), this polemic panorama has activated a reworking of the neutrino physics to establish any definite conclusion about the detailed aspects of the interior current supernova processes from the observed data (Sawyer, 1988; Lattimer, 1988). Whatever the answer to the above points may be, now it is a well-established fact that a shock wave is formed as the result of the infall of the outer envelopes upon a more compact (halted or rebounding) inner core and this view is independent of the possible role played by the shock on the supernova outcome.

Within this context the aim of this work is to stimulate discussion over some points which have recently been raised (Herrera and Núñez, 1987, 1991; Barreto et al., 1989, 1991) and which we believe deserve some attention. They concern the following questions:

(a) Where does the compact remnant separates from the expanding matter of the ejected mantle in supernova outburst?

(b) Why does the outer mantle bounce?

In both, prompt and long-term mechanism, the shock originating near the sonic point (we shall call it the original shock) and propagating outward counteract the infall and thus cause ejection of a bulk of matter. A proto-neutron star is left behind as a result, in the form of a compact remnant. The main difference between the two proposed descriptions resides in the fact that in the long-term mechanism the shock stalls and it is somehow revitalized leading afterwards to an explosion. The separation between the inner core and the expanding and ejected outer mantle for this pcture takes place on the original shock.

We have considered in recent works (Herrera and Núñez, 1987, 1991; Barreto et al.,

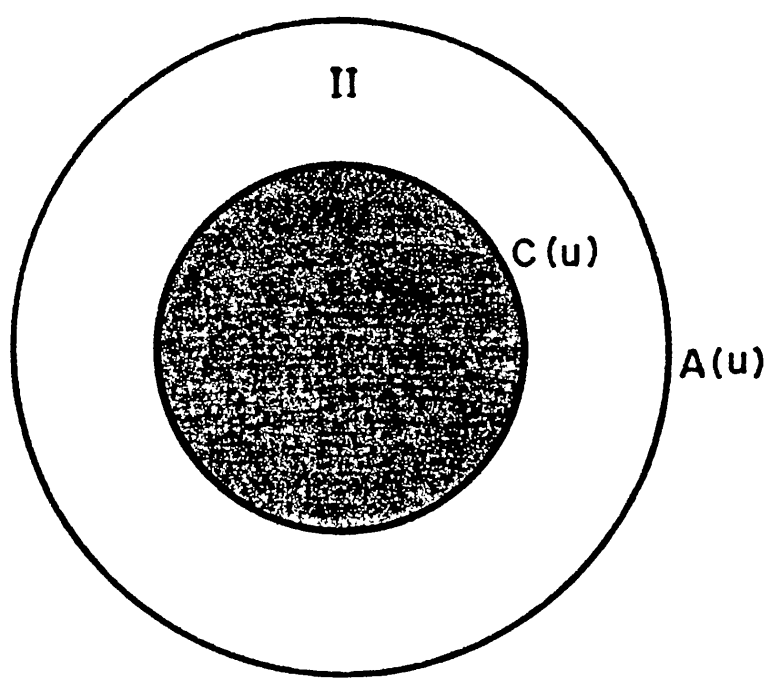

Fig. 1. The two-region model sphere considered throughout this work. Region I, the inner core, is enclosed by a shock surface $C(u)$. The outer mantle completes the sphere of radius $A(u)$. 
1991) a general relativistic radating fluid sphere divided in two regions by a shock wave front (Figure 1). At either side a physically reasonable equation of state is chosen and the luminosity is assumed to be a Gaussian-like pulse which carries out a fraction of the total initial mass. In the first two papers (cf. Herrera and Núñez, 1987, 1991), we have assumed the free streaming-out limit, i.e., the flux emission is represented by a null fluid. Variations in the shape of the luminosity profiles on the shock front and on the boundary surfaces introduce time delays for emission and absorption on these surfaces.

In Figure 2 it is sketched the evolution of both the radius of the sphere (the boundary surface) and the shock wave front. As it can be appreciated from this figure the position

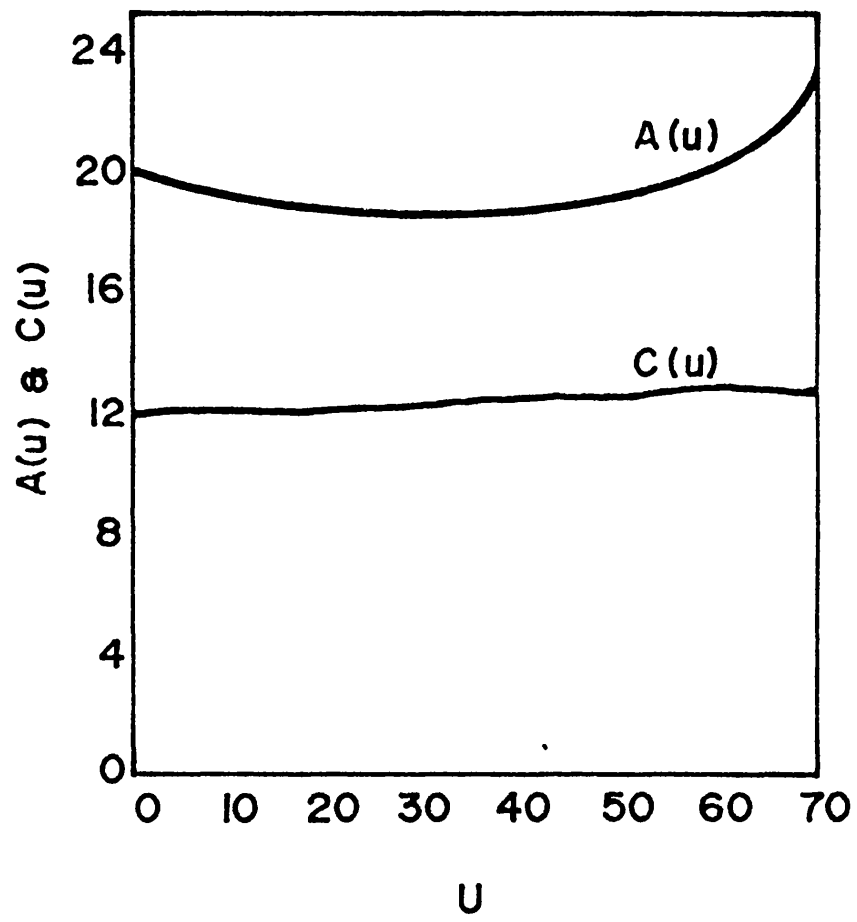

Fig. 2. Time evolution of $A(u)$ and $C(u)$ for the exploding sphere in the 'free streaming-out limit' for the radiation flux.

of the shock remains almost constant although the boundary surface bounces. It is apparent from Figure 3 that the outer-matter shells bounce earlier than inner ones.

This bouncing scheme is maintained until the shock surface is reached. Moreover, during the bounce of the mantle the shock wave separating the compact inner core from the outer mantle fades out (Figure 4); therefore, the early discontinuity surface bounding the core evolves into a boundary of the static and homogeneous remnant. The origin of the peculiar bouncing of the mantle is to be found in the assumption of free streaming-out radiation field propagating outward through the outer mantle with a Tolman VI-like equation of state. In fact, Herrera et al. (1980) originally found this scheme of bouncing for a Tolman VI-like sphere assuming as well a free streaming-out radiation field. Recent calculations (Barreto et al., 1989, 1991) for the same Tolman VI 


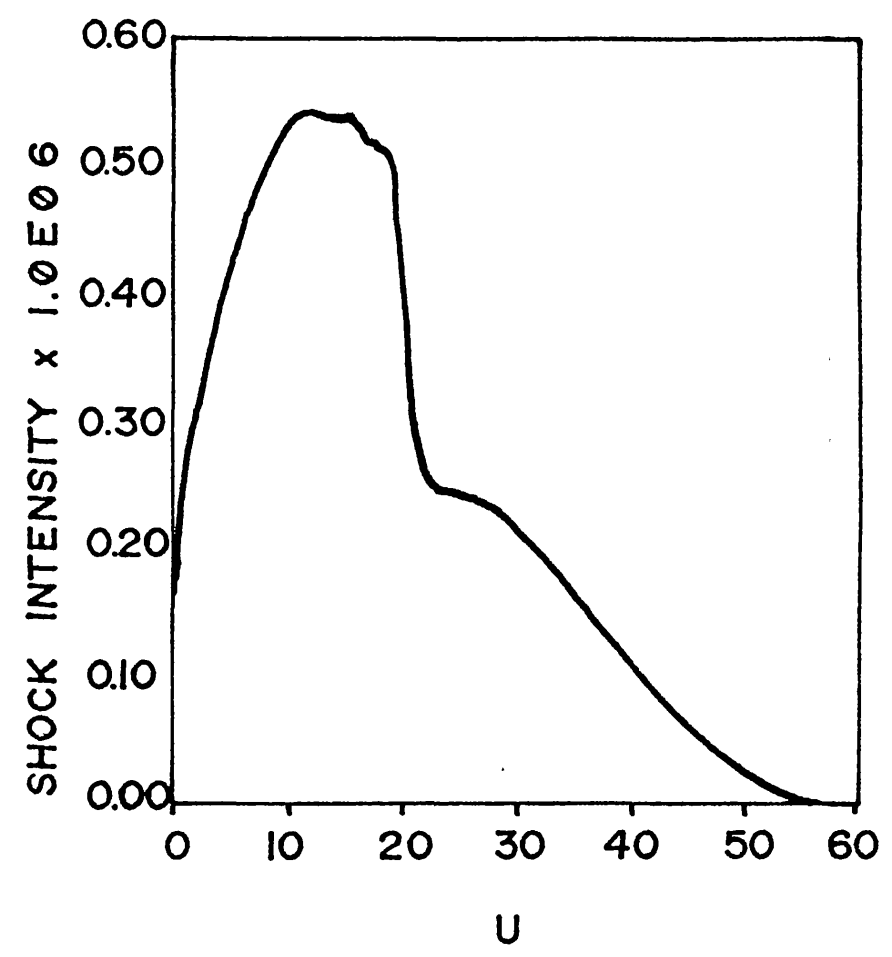

Fig. 3. Time evolution of the intensity of the shock, $\mathscr{I}=P_{I I}-P_{I}$, in the free streaming-out limit case.

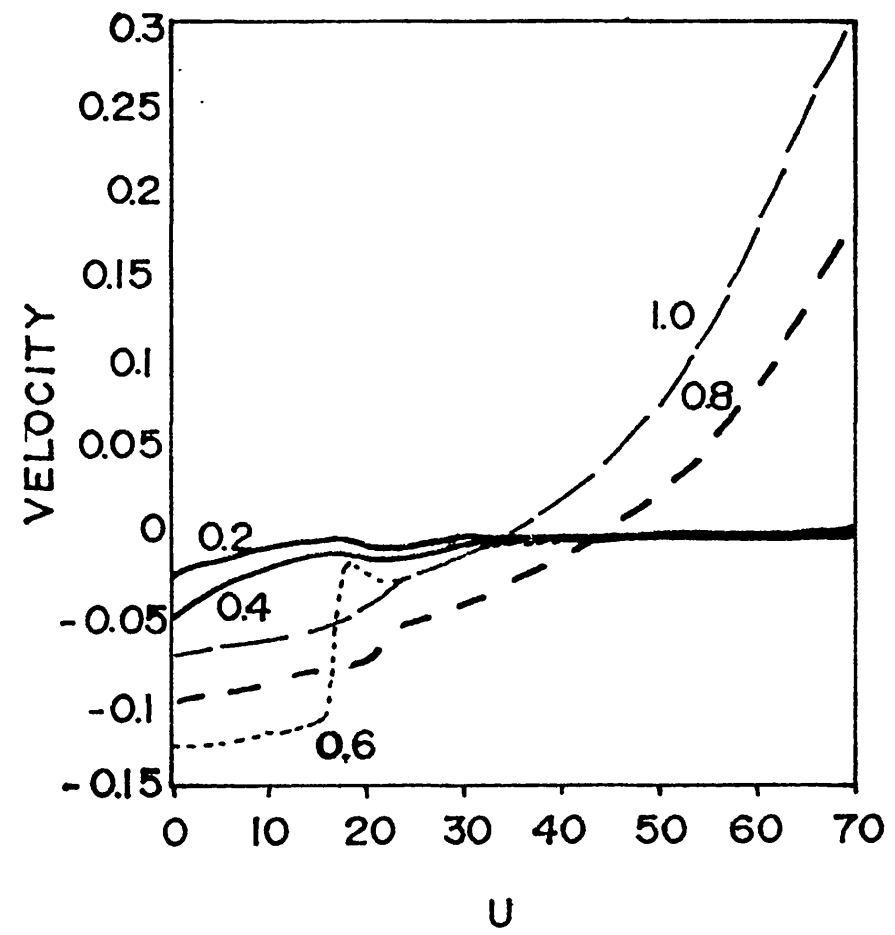

Fig. 4. The evolution of mass velocity in the free streaming-out limit is monitored at four different fixed mass shells. Labels corresponds to ratios $r / a_{0}=0.2,0.4,0.6,0.8,1.0$. 
model, but in the diffusion limit approximation, yield the opposite picture for the bouncing, i.e., inner shells bounce earlier than the outer ones.

The bouncing of the mantle could be though to be produced by its progressive inward stiffness variation and not by the outward propagation of a shock. Furthermore, as a given shell spring back, a rarefaction zone is created which facilitates the adjacent inner one to bounce, overcoming the gravitational attraction. This sequence is repeated until the fading shock is reached. The shock does not appear to be directly responsible for the expulsion of the outer envelopes of the sphere material, but it is closely related to it. It is also well established that a deeper recession of the shock front is related to a stronger explosive ejection. This peculiar recession-explosion association and the general hydrodynamic scenario obtained recall those situations where the long-term mechanism leads to viable supernova outcome. However, we do not find any revival of the original shock. Should the picture above turns out to be a suitable description of a supernova event, then strong shock fronts will no longer be required to explain the ejection of the outer envelope nor will it be necessary to look for the revitalization of stalled shocks. Moreover, the surface which delimits the remnant is the shock itself, once it has faded out and transformed into a boundary. If the interface propagates outward, the inner core grows up by accretion of the outer mantle onto it, and, thereby, this would hinder the appropriate ejection conditions to settle down.

In the models considered so far, we have assumed the free streaming-out limit to be valid. However, in certain situations of stellar collapse, diffusion processes might play important roles. For example, the opacity of collapsing matter for neutrinos/photons will give rise to diffusion processes associated with radiative thermal conduction (cf. Kazanas, 1978; Mihalas and Mihalas, 1984). Also, as it has been recently reported, the low mean energy of the detected neutrinos $(\cong 10 \mathrm{MeV})$ from the supernova 1987A and

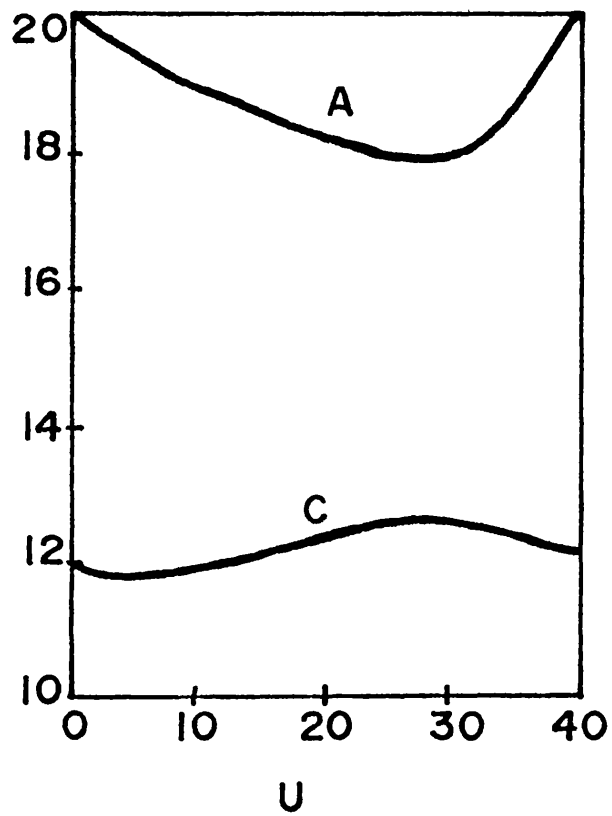

Fig. 5. Evolution of $A(u)$ and $C(u)$ in the diffusion limit for the radiation flux. 
the long duration of the burst, attest to the diffusion limit of the neutrinos from the lepton-rich proton-neutron star (Lattimer, 1988).

Figure 5 displays the evolution of the boundary surface and the shock front for a composite sphere where diffusion limit is assumed throughout, except at abovementioned surfaces. As well as in the free streaming-out case, the shock does not reach the boundary of the distribution. The shock expands and later it recedes and, during this period, the boundary surface bounces expanding with an increasing velocity. It is found that the expansion of the shock enhances the expulsion of the outer matter shells. This piston-like effect, that makes the shock drive out the outer mantle, can also be observed in Figure 6 where velocity profiles, recorded at different mass shells, are

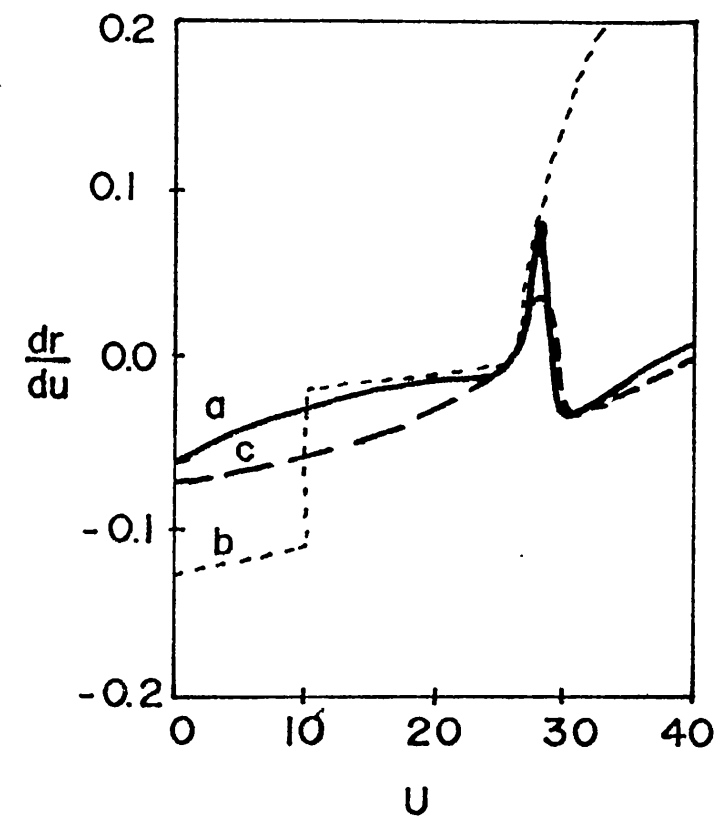

Fig. 6. Evolution of the mass velocity in the diffusion limit monitored at different fixed mass shells. Curves are labeled by the corresponding ratios $r / a_{0}=0.2,0.6,1.0$.

displayed. It is worth to be noted that the bounce at every mass shell occurs at the same retarded time $u=$ const. Therefore, as it is seen by a distant observed $(u \cong t-r)$, inner shells bounce earlier than the outer ones. This result reveals a deep difference between the free streaming-out and the diffusion regimes. In order to understand further the picture emerging from the modeling performed, use has been made of a generalisation of the concept of adiabatic index for non-adiabatic systems.

This new variable measures the stiffness of the equation of state for a radiant fluid. It is defined by

$$
\Gamma_{\mathrm{NA}}=\frac{\mathrm{d} \ln \Pi}{\mathrm{d} \ln E}
$$


where $\Pi$ and $E$ are the total flux of momentum in the direction of contraction and the total energy flux density, respectively. Both, $\Pi$ and $E$ are measured by a suitable defined Minkowskian observer (see Barreto et al., 1989; for details).

The stiffness for both the inner core and the outer mantle can be readily observed in Figures 7 and 8 . The non-adiabatic index for the material is greater at the core side of

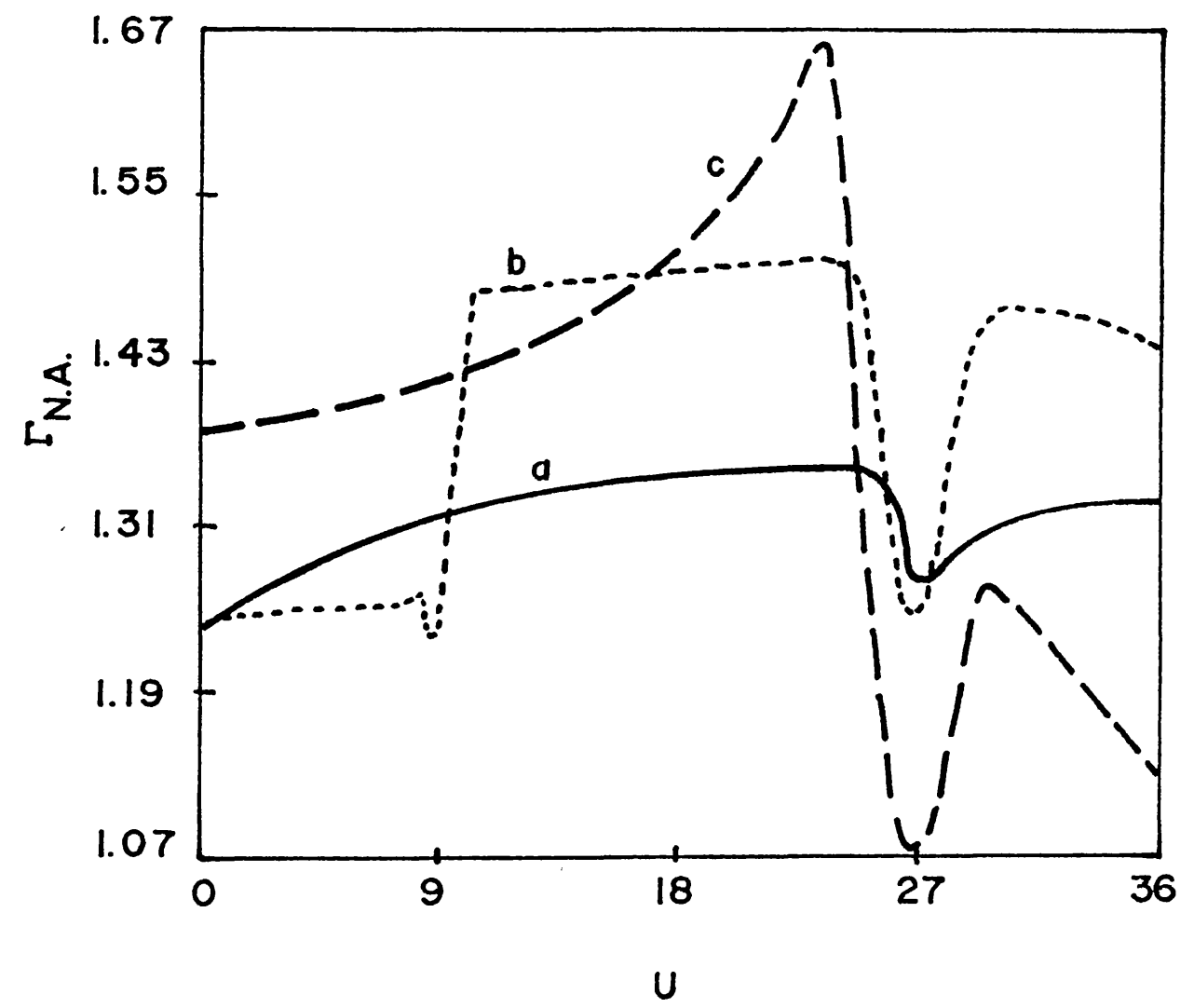

Fig. 7. Evolution of the non-adiabatic index measured at different fixed mass shells. Curves are labeled by the corresponding ratios $r / a=0.2,0.6,1.0$.

the shock than at the mantle side (Figure 9). It is worth noting that the emission of radiation soften the material sphere and, therefore, the NA-index diminishes (Figure 7). Moreover, after the emission of the radiation pulse, core inner shells asymptotically collapse to the hydrostatic limit. It is important to note from curves c-d in Figure 8, how well-defined zones, i.e., stiff core and soft mantle, emerge after the emission of the radiation pulse.

Let us now, on the basis provided by our modeling, try to set up an intelligible scheme in order to understand the mantle bouncing effect. The very basis of this effect is clearly brought out by Figure 8. Observe the increasing, before bouncing of the NA-index at outer regions and its subsequent 'relaxation' after the bounce. This stiffening-softening of the outer mantle matter, clearly leading to an expansion, is preceded by the appearance of a minimum for the NA-index at the mantle side of the shock. The composite sphere, therefore, 'breaks' at the shock front. As a result of these two effects 


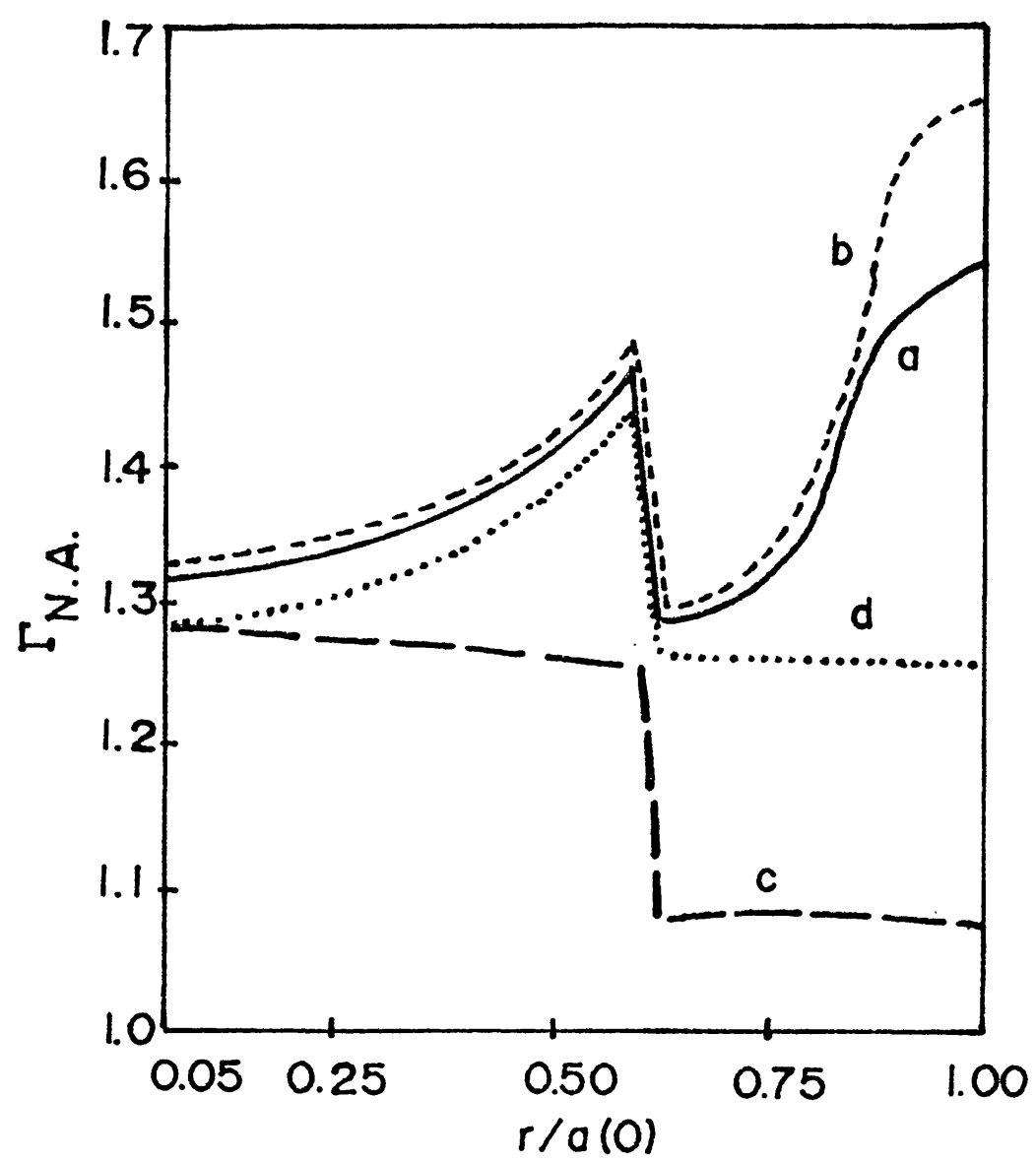

Fig. 8. The non-adiabatic index as a function of the dimensionless space-like coordinate $r / a(0)$. Curves are labeled by the corresponding radiation times, $u=20,24,28,30$.

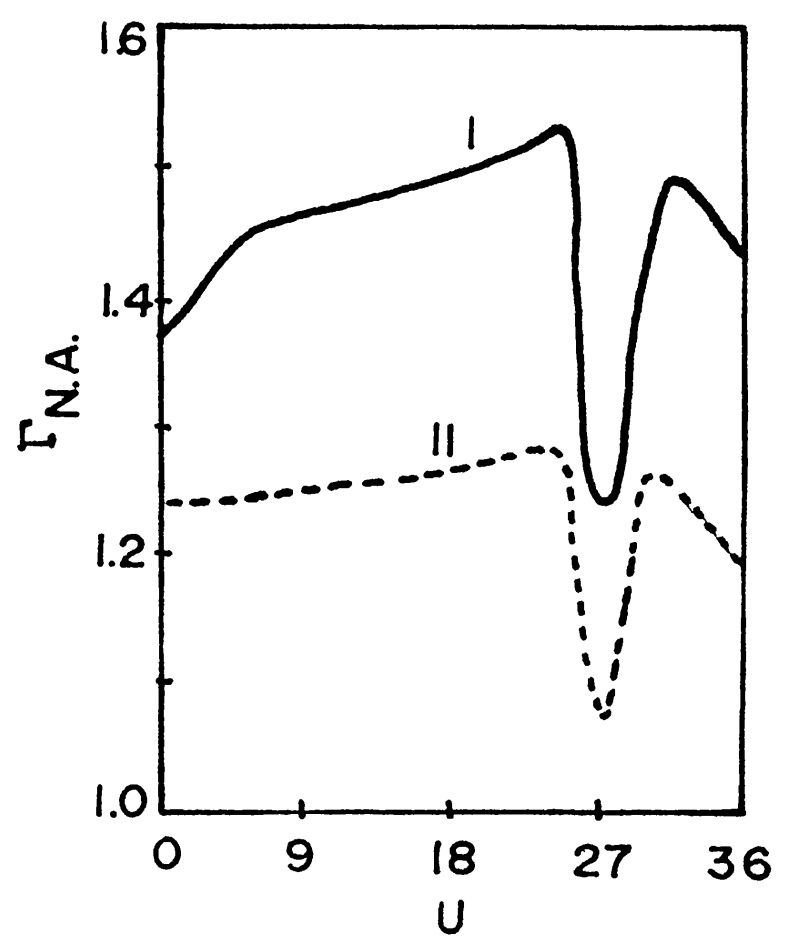

Fig. 9. The evolution of the non-adiabatic index at the shock front. 
(stiffening-softening and breaking) we shall have a compact homogeneous remnant and an ever-expanding mantle. This picture is valid for both radiation limits.

Thus in the context of a consistent general relativistic framework, and adopting simple, but not extremely unphysical equations of state, we are able to set up a hydrodynamical description for the bouncing mechanism where a strong shock wave that blows up the outer mantle is not present. We are perfectly aware of the fact that a long way has to be covered before to extract definite conclusion concerning the collapse of a real massive star. Nevertheless, the coherence of the scheme commented above is striking enough as to suggest that possibility.

Before concluding we would like to remark that, as far as we know, the only observable quantity entering into a 'real' gravitational collapse is the luminosity profile. Therefore, it seems reasonable to adopt the approach we have followed in closing our modeling providing such profiles. In the same order of ideas, it should be emphasized that ultradense matter is not 'available' in any earth laboratory. And all 'known' equations of state for these materials, independently of how 'elaborated' is the microphysics they use, emerge from not very well justified extrapolations and speculations.

\section{Acknowledgements}

This work has been partially supported by the Consejo de Desarrollo Científico Humanístico y Technológico de la Universidad de Los Andes, under project 379-89-05.

\section{References}

Aglietta, M. et al.: 1987, Europhys. Letters 3, 1315.

Arnett, W. D.: 1987, Astrophys. J. 319, 136.

Baron, E., Bethe, H. A., Brown, G. E., Cooperstein, J., and Kahana, S. H.: 1987, Phys. Rev. Letters 59, 736.

Barreto, W., Herrera, L., and Santos, N. O.: 1989, Astrophys. J. 344, 158.

Barreto, W., Herrera, L., and Núñez, L.: 1991, Astrophys. J. (to appear).

Bethe, H. A. and Wilson, J. R.: 1985, Astrophys. J. 295, 14.

Bethe, H. A., Brown, G. E., Applegate, J., and Lattimer, J. M.: 1979, Nucl. Phys. A324, 487.

Bionta, R. M. et al.: 1987, Phys. Rev. Letters 58, 1494.

Bruenn, S. W.: 1987, Phys. Rev. Letters 59, 938.

Burrows, A.: 1987, Astrophys. J. 318, L57.

Colgate, S. A. and Johnson, H. J.: 1960, Phys. Rev. Letters 5, 235.

De Rújula, A.: 1987, Phys. Letters 193B, 514.

Herrera, L. and Núñez, L.: 1987, Astrophys. J. 319, 868.

Herrera, L. and Núñez, L.: 1990, Astrophys. J. 364, 212.

Herrera, L., Jiménez, J., and Ruggeri, G.: 1980, Phys. Rev. D22, 2305.

Hillebrandt, W., Höflich, P., Kafka, P., Müller, E., Schmidt, H. U., and Truran, J. W.: 1987, Astron. Astrophys. 180, L20.

Hirata, K. et al.: 1987, Phys. Rev. Letters 58, 1490.

Kahana, S. H., Cooperstein, J., and Baron, E.: 1987, Phys. Letters 196B, 256.

Kazanas, S. H.: 1978, Astrophys. J. 222, L109.

Lattimer, J. M.: 1988, Nucl. Phys. A478, 199c.

Mihalas, D. and Mihalas, B.: 1984, Foundations of Radiations Hydrodynamics, Oxford University Press, Oxford, p. 467. 
Sawyer, R. F.: 1988, Astrophys. J. 328, 691.

Stella, A. and Treves, A.: 1987, Astron. Astrophys. 185, L5.

Trimble, V.: 1988, Rev. Mod. Phys. 60, 859.

Wilson, J. R.: 1985, in J. Le Blanc and R. Bowers (eds.), Numerical Relativity, Jones and Bartlett, Boston. 